\title{
Rate Representation of Tones in Noise in the Inferior Colliculus of Decerebrate Cats
}

\author{
Ramnarayan Ramachandran, ${ }^{1}$ Kevin A. Davis, and Bradford J. May ${ }^{2}$ \\ ${ }^{1}$ Department of Biomedical Engineering and $\mathrm{Center}$ for $\mathrm{H}$ earing and Balance, Johns $\mathrm{H}$ opkins University, \\ Baltimore, M D 21205 USA \\ ${ }^{2}$ Department of Otolaryngology-H NS and Center for Hearing and Balance, Johns H opkins University, \\ Baltimore, M D 21205 USA
}

Received: 1 May 2000; Accepted: 18 May 2000; O nline publication: 29 August 2000

\begin{abstract}
Neurons in the central nucleus of the inferior colliculus (ICC) of decerebrate cats show three major response patterns when tones of different frequencies and sound-pressure levels (SPLs) are presented to the contralateral ear. The frequency response maps of type I units are uniquely defined by a narrow excitatory area at best frequency (BF: a unit's most sensitive frequency) and surrounding inhibition at higher and lower frequencies. As a result of this receptive field organization, type I units exhibit strong excitatory responses to $B F$ tones but respond only weakly to broadband noise (BBN). These response characteristics predict that type I units are well suited to encode narrowband signals in the presence of background noise. To test this hypothesis, the dynamic range properties of ICC unit types were measured under quiet conditions and in multiple levels of continuous noise. As observed in previous studies of the auditory nerve and cochlear nucleus, type I units showed upward threshold shifts and discharge rate compression in background noise that partially degraded the dynamic range properties of neural representations at high noise levels. Although the other two unit types in the ICC showed similar trends in threshold shift and noise compression, their ability to encode auditory signals was compromised more severely in increasing noise levels. When binaural masking effects were simulated,
\end{abstract}

Correspondence to: Bradford J. May • Department of O tolaryngologyH NS • Johns H opkins U niversity • 720 Rutland Avenue 505 Traylor Research Building • Baltimore, MD 21205. Telephone: (410) 9553162; fax: (410) 955-1299; e-mail: bmay@bme_jhu.edu only type I units showed an enhanced representation of spatially separated signals and maskers that was consistent with human perceptual performance in independent psychoacoustic observations. These results support the interpretation that type I units play an important role in the auditory processing of narrowband signals in background noise and suggest a physiological basisfor spatial factors that govern signal detection under free-field listening conditions.

Keywords: auditory system, binaural hearing, signal encoding, background noise

\section{INTRO DUCTIO N}

Neurons in the central nucleus of the inferior collicuIus (ICC) exhibit three major response types when single-unit responses are recorded in unanesthetized decerebrate cats (Ramachandran et al. 1999). Figure 1 (left panels) shows typical frequency response maps for each unit type. The response maps of type I units are distinguished by a receptive field organization that combines a narrowly tuned I-shaped area of excitation at frequencies around best frequency (BF: the most sensitive frequency) and flanking regions of inhibition at higher and lower frequencies. Type $V$ units produce frequency response maps that display a broadly tuned V-shaped excitatory area with no signs of inhibition. Type 0 units have frequency response maps that are dominated by inhibition except for an $\mathrm{O}$-shaped island of excitation at frequencies near BF and sound-pressure levels near threshold.

The rate-level functions in Figure 1 (right panels) offer an alternative method for visual izing the strength 

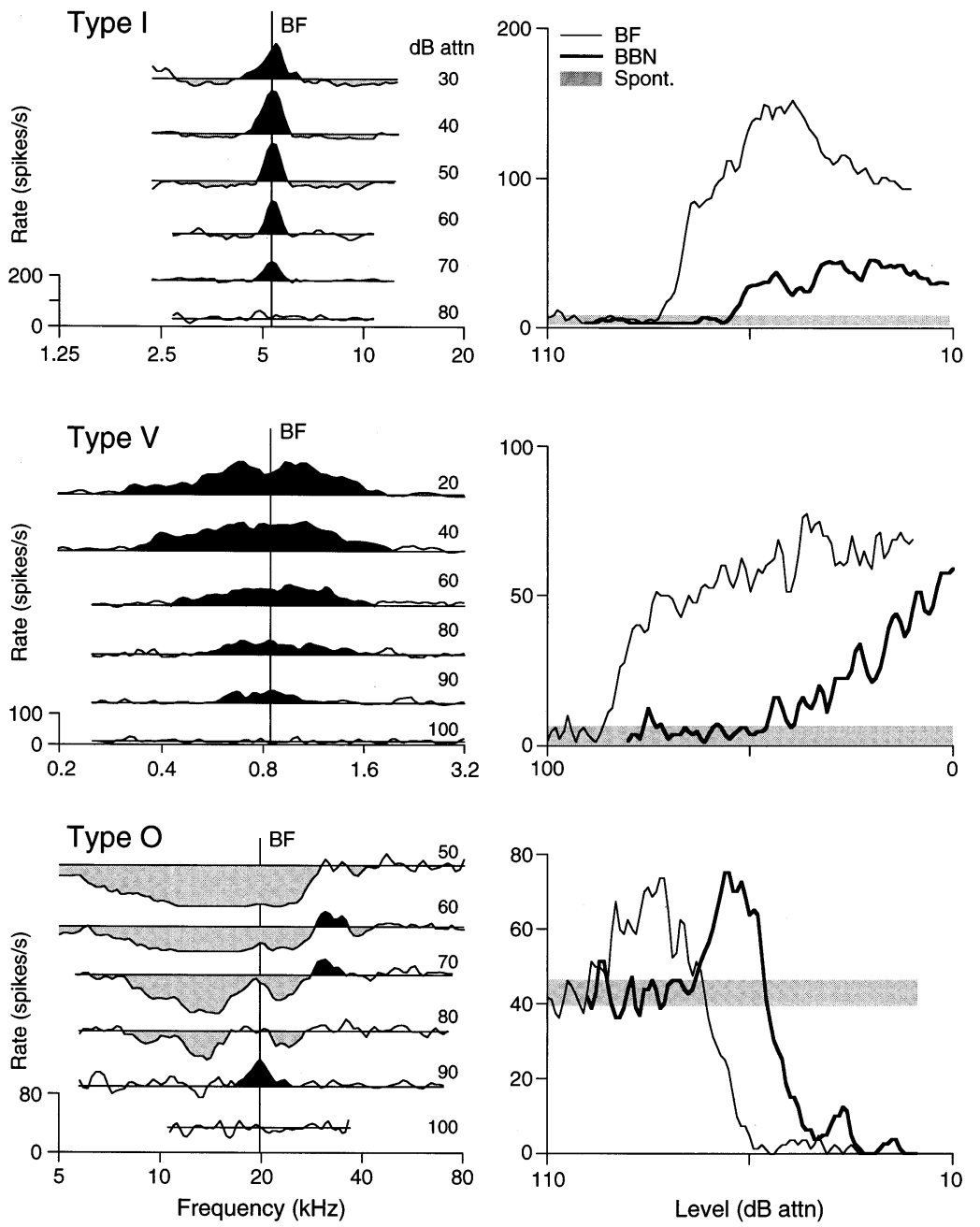

FIG. 1. M onaural response properties of a representative type I unit (unit number $2.10, \mathrm{BF}=5.4 \mathrm{kHz}$, experiment date: 01/26/1998), type $V$ unit (unit number $4.01, \mathrm{BF}=1.2 \mathrm{kHz}$, experiment date: $09 / 20$ / 1996), and type 0 unit (unit number $1.20, B F=20$ $\mathrm{kHz}$, experiment date: 04/30/1997). Combinations of frequency and level that elicited excitatory (inhibitory) discharge rates are identified by black (gray) regions on frequency response maps in the left panels. The scale that was used to plot response magnitude is indicated by the axis at the bottom left of each map. Tone levels are specified in $\mathrm{dB}$ attenuation by numerical labels along the right margin of each map. Rate-level functions for BF tones and bursts of broadband noise are shown in the right panels. Gray shading encompasses the range of spontaneous rates that were recorded during the collection of rate-level data. of on-BF inhibition that is typical for each ICC unit type. Type I and $V$ units show only excitatory responses when BF tones or broadband noise bursts are presented at high stimuluslevels; the resulting monotonic or slightly nonmonotonic rate-level functions suggest that on-BF inhibition is relatively weak for these units. By contrast, the rate-level functions of type 0 units are characterized bya pronounced nonmonotonicity. This strong on-BF inhibition leads to al most complete cessation of type $O$ discharge rates at high stimulus levels.

Variations in the strength and frequency organization of the excitatory and inhibitory inputs to ICC neurons (Ramachandran et al. 1999), as well as concomitant differences in binaural response properties (Davis et al. 1999; Ramachandran and May 1999a, b), suggest that ICC response types may reflect a higherorder manifestation of the unique signal processing attributes of the auditory brainstem nuclei. Level-tolerant excitator y responses for contralateral tones, inhibitory responses to ipsilateral stimulation, and binaural inhibitory interactions have been cited as evidence that typel units receive ascending inputs from neurons in the lateral superior olive (LSO). By contrast, type $\mathrm{V}$ and type $\mathrm{O}$ units share monaural and binaural response properties with neurons in the medial superior olive (MSO) and the dorsal cochlear nucleus (DCN), respectively.

The existence of ICC unit types with response properties matching those of the principal brainstem neurons suggests that ascending auditory pathways may remain functionally segregated at higher levels of processing. Given this interpretation, physiological characterization of single-unit responses in the ICC not only can reveal the dominant sources of input to the midbrain but also can evaluate transformations in the quality of these ascending representations at the level of the inferior colliculus. O ne aspect of auditory information processing that hasinterested physiologistsand psychophysicists for many years is how acoustic signals are made less detectable by competing sounds. In particular, the neural encoding of BF tones in background noise has been the subject of detailed electrophysiological measures in the auditory periphery, brainstem, and inferior colliculus (auditory nerve: Rhode et al. 
1978; Costalupes et al. 1984; Gibson et al. 1985; cochlear nucleus: Gibson et al. 1985; Rhode and Greenberg 1994; inferior colliculus: Rees and Palmer 1988).

Rhode and Greenberg (1994) have noted that mechanisms for lateral suppression and inhibition in the cochlear nucleus potentially enhance the rate representation of auditory signals under noisy background conditions, presumably by sharpening frequency selectivity. The present study recorded single-unit responses in the ICC of decerebrate catsunder quiet conditions and in various levels of continuous broadband noise to evaluate the hypothesis that the lateral inhibition of type I units also reflects an auditory specialization that is well-suited for processing signals in noise. A signal detection analysis of the electrophysiological results obtained in these experiments suggests that type I units provided a more sensitive rate representation of auditory signals in background noise than auditory ner ve fibers, cochlear nucleusneurons, or ICC units with other patterns of receptive field organization.

\section{METHODS}

All surgical and recording procedureswere performed under guidelines of The Johns H opkinsU niversity Animal Care and U se Committee.

\section{Surgical preparation}

Adult male cats with clean external ears and middle ears free of infection were anesthetized with xylazine ( $2 \mathrm{mg}, \mathrm{im}$ ) and ketamine (initial dose $100 \mathrm{mg}$, im; supplemental doses $15 \mathrm{mg}$, iv). Atropine sulphate ( 0.1 $\mathrm{mg}$, im) was given to control mucous secretions. The cephalic vein was cannulated to allow intravenous injections of fluids, and a tracheotomy was performed to facilitate quiet breathing. The cat's core temperature was maintained at $39^{\circ} \mathrm{C}$ by a regulated heating pad. A midline incision was made over the skull and the temporalis musclesreflected. The skull wasopened over the parietal cortex and the cat was made decerebrate by aspiration of the thalamus under visual control. Anesthesia was discontinued and the completeness of decerebration was established by the lack of voluntary movements during subsequent electrophysiological recording. Both ear canals were transected to accept the hollow ear bars that delivered closed-field acoustic stimuli. The tympanic bullae were vented with polyethylene tubing to prevent static pressure build-up in the middle ear. A second fenestration was made over occipital cortex and the dorsal tectum was exposed by cortical aspiration and partial removal of tentorium. Localization of recording sites was determined online by the sequence of BFs along electrode tracks and by the prevalence of ICC response types that were identified in previous experiments (Ramachandran et al. 1999; Davis et al. 1999). At the conclusion of the recording session (48-72 hours after the start of surgery), cats were euthanized with an overdose of sodium pentobarbital ( $125 \mathrm{mg}$, iv). Some subjects were perfused to allow histological confirmation of the completeness of the decerebration and the location of recording sites.

\section{Recording protocol}

Electrophysiological recordings were made in a soundattenuating chamber. Stimuli were delivered via electrostatic speakers that were coupled to the hollow ear bars; the closed-field acoustic system produced signals with minimal variation in stimulus level either across frequencies ( $\pm 5 \mathrm{~dB}$ from $40 \mathrm{~Hz}$ to $40 \mathrm{kHz}$ ) or between ears $( \pm 2 \mathrm{~dB})$. All binaural tests were performed below stimulus levels that were capable of stimulating the contralateral ear via conduction through the stereotaxic apparatus (i.e., acoustic crosstalk; Gibson 1982).

Single-unit activity was recorded with platinumiridium microelectrodes. The electrode signal was amplified 10,000-30,000 $\times$ and lowpass filtered at 5 $\mathrm{kHz}$. A variable threshold Schmitt trigger was used to discriminate action potentials from background activity. Digitized spike trains were created for subsequent analysis by recording spike times relative to stimulus onset. Rate-level functions were obtained with 200-ms tone bursts that were presented at a presentation rate of 1 burst/s. Tone-driven rates were computed by counting spikes that occurred during the stimulus-on interval (200 ms in duration, beginning $10 \mathrm{~ms}$ after stimulus onset); spontaneous rates were measured by counting spikes during the final 400 ms of the stimulusoff interval.

Search stimuli (50-ms tones or noise bursts) were presented as electrodes and advanced dorsoventrally through the inferior colliculus. Entry into ICC was indicated by a reversal in the progression of BFs relative to single units and background activity in the dorsal cortex of the inferior colliculus (Merzenich and Reid 1974; Aitkin et al. 1975). When an ICC unit was encountered and successfully isolated, it was tested with a series of tone bursts that swept in frequency across a four-octave range centered on unit BF. The frequency sweeps were presented at levels of 10 and $40 \mathrm{~dB}$ re threshold. The resulting partial frequency response maps were sufficient to identify ICC unit types based on patterns of inhibition that were described in Figure 1 (Ramachandran et al. 1999). 


\section{Data collection}

Rate-level functions were obtained by presenting a series of fixed-frequency (BF) tone bursts that varied in level over a 100-dB range; the lowest level in the series was placed approximately $10 \mathrm{~dB}$ below the unit's threshold, as defined below. BF tones were presented in four stimulus contexts: (1) monaural presentations to the contralateral ear in quiet, (2) monaural (contralateral) presentations in continuous broadband noise, (3) binaural presentations in quiet, and (4) binaural tones in binaural background noise. Rate-level functions were sampled in 10-dB increments with ten repetitions to obtain statistical measures of the rate representation of each stimulus level. In some instances, rate-level functions were obtained with 1-dB resolution without repeating levels to produce a more detailed description of a neuron's dynamic range properties. Data collection for tone presentations in background noise began five seconds after noise onset to allow discharge rates to stabilize. O n occasion, units showed a slow rate of noise adaptation that progressed throughout the collection of the rate-level function; these long-term adaptation effects did not appear to be more prevalent for any particular response class.

Binaural stimuli simulated free-field interaural level difference (ILD) cues to investigate the influences of sound location on the representation of signals in noise. ILD effects were characterized under quiet conditions using a contralateral tone with fixed level ( 10 $\mathrm{dB}$ re threshold) and ipsilateral tones that swept in level from $20 \mathrm{~dB}$ below the contralateral tone $(+20$ $\mathrm{dB} I L D)$ to $20 \mathrm{~dB}$ above the contralateral tone $(-20$ $\mathrm{dB}$ ILD). Responses to binaural tonesin binaural noise were studied by fixing the ILD of the tone signal at $+20 \mathrm{~dB}$, fixing the noise level in the contralateral ear at $10 \mathrm{~dB}$ re threshold, and varying the noise level in the ipsilateral ear to produce ILDs from +20 to -20 $\mathrm{dB}$ in $10-\mathrm{dB}$ steps.

\section{RESU LTS}

Single-unit responses were recorded from 118 ICC neurons in 14 cats. The present analysis was based on average discharge rate and, therefore, was limited to units that showed sustained responses to BF tones. Strictlyonset responses are relativelyuncommon in the ICC of decerebrate cats [Ramachandran et al. (1999) observed 12 onset units in a sample of 146 ICC units]. Additional stimulus protocols were performed in conjunction with this study to maximize the collection of data from individual subjects.
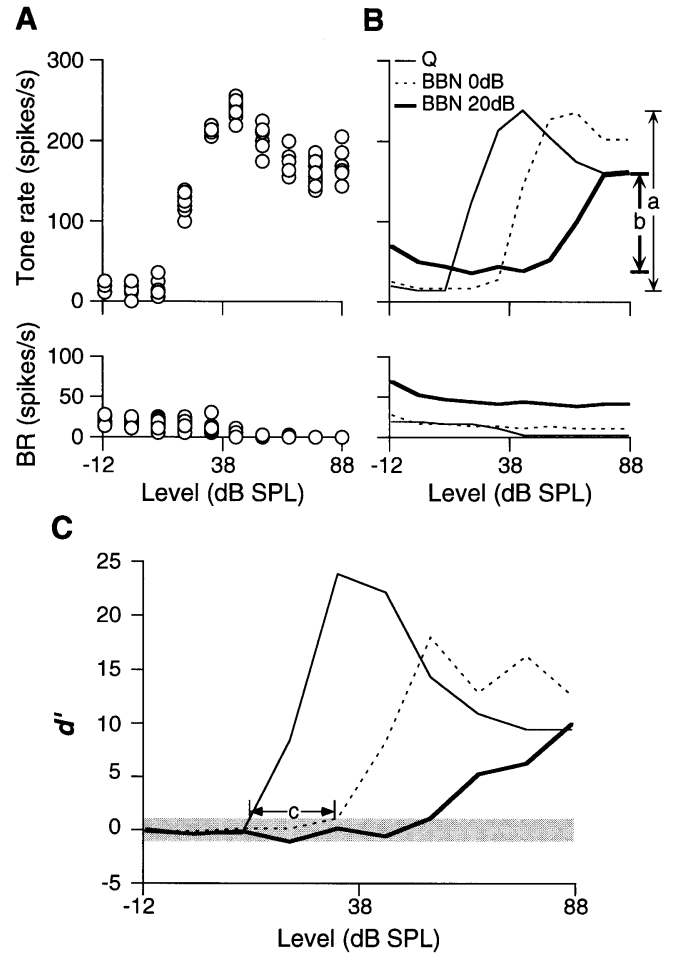

FIG . 2. Statistical methods for measuring rate-level functions for $B F$ tones under quiet conditions and in continuous background noise (data from type I unit number $7.10, \mathrm{BF}=36.3 \mathrm{kHz}$, experiment date: 08/25/1997). A. Tone-driven rates during stimulus-on intervals (upper panel) and their corresponding background rates during stimulus-off intervals (lower panel). Responses were sampled 10 times at each stimulus level. These data were obtained under quiet conditions. B. Average rate-level functions and background rates for BF tones in quiet and in two levels of continuous background noise. The ratelevel function in quiet (thin line) represents the average of data shown in A. C. $\mathbf{d}^{\prime}$ functions based on differences between the tone-driven and background rates in $\mathbf{B}$. See text for a description of the signal detection methods that were used in these calculations. The grayshaded region identifies tone levels that produced $d^{\prime}$ values between \pm 1 ; from a signal detection perspective, the presentations of these stimuli cannot be detected in the neuron's discharge rates.

Effects of background noise on rate-level functions of type I units

Figure 2 illustrates the data collection methods that were used to characterize the rate encoding of $\mathrm{BF}$ tone levels by ICC neurons. The data shown in Figure 2 were obtained from a type I unit. In Figure 2A, the plots of driven ratesduring the tone-on interval (upper panel) and background rates (BRs) during the toneoff interval (lower panel) indicate the range of responses obtained when each stimulus level in the $100-d B$ series was repeated ten times. These data were obtained under quiet conditions; therefore, the unit's $B R$ is its spontaneous activity. In Figure 2B, the unit's mean driven rates and $B R$ sare compared for recording under quiet conditions (thin line), in continuous broadband noise with a 0-dB spectrum level (dashed line), and in a noise spectrum level of $20 \mathrm{~dB}$ (bold 
line). One prominent noise effect was a shift in the dynamic range of the rate-level function to higher tone levels. The second effect was rate compression, a decrease in the range of rate changes that potentially encode stimulus levels. Rate compression results from an increase in background rates because of the unit's sustained noise-driven activity ( lower panel) and a concomitant decrease in maximum tone-driven rates that most likely results from a combination of noise inhibition and short term adaptation (upper panel).

Statistical analyses were performed on discharge rates to evaluate the effects of noise-induced dynamic range shifts and rate compression from a signal detection perspective (Green and Swets 1974). The discriminability index $\left(d^{\prime}\right)$ was calculated using eq. (1):

$$
d^{\prime}=\frac{\mu_{\mathrm{t}+\mathrm{B}}-\mu_{\mathrm{B}}}{\sqrt{\sigma_{\mathrm{t}+\mathrm{B}}^{2}+\sigma_{\mathrm{B}}^{2}}}
$$

where $\mu_{t+\mathrm{B}}$ is a unit's mean driven rate for the ten repetitions of a tone level in a background of either quiet or noise, and $\mu_{\mathrm{B}}$ is the unit's mean background rate during the corresponding stimulus-off intervals. $\sigma_{\mathrm{t}}+\mathrm{B}$ and $\sigma_{\mathrm{B}}$ are the standard deviations of those responses. This form of eq. (1) assumes that discrimination is based on two observation intervals ( stimuluson and stimulus-off) and that the decision variable is the difference in discharge rates between the two inter vals. By convention, a d' value of \pm 1 was used as the criterion that the tone presentation produced a detectable change in discharge rate relative to background activity. Figure $2 \mathrm{C}$ plots $\mathrm{d}^{\prime}$ values that were derived from the discharge rates shown in Figure 2A. As was seen for average discharge rates, $d^{\prime}$ values at fixed sound-pressure levels decreased in the presence of noise; consequently, the dynamic component of the signal detection function was translated to higher levels. This shift in the dynamic range of the rate response predicts that low levels of tone are less detectable in background noise, which is a well-established psychoacoustic masking phenomenon.

Dynamic range shifts were quantified by calculating the difference between signal detection thresholds in quiet conditions and in various levels of background noise (arrows labeled $\mathrm{c}$ in Fig. $2 \mathrm{C}$ ). Figure $3 \mathrm{~A}$ plots shifts in the tone detection thresholds of the representative type I unit as a function of noise spectrum level. The unit's tone thresholds were not affected by noise spectrum levels less than $0 \mathrm{~dB}$ because these noise levels fell below the unit's noise threshold and, therefore, failed to elicit inhibitory or adaptation effects. At higher noise spectrum levels, the detection threshold shifted to progressively higher tone levels with each increase in the level of background noise. The noiseinduced threshold shifts of type I units were linear over a wide range of spectrum levels (dashed line in Fig. 3A). Figure 3B shows the threshold shifts that were
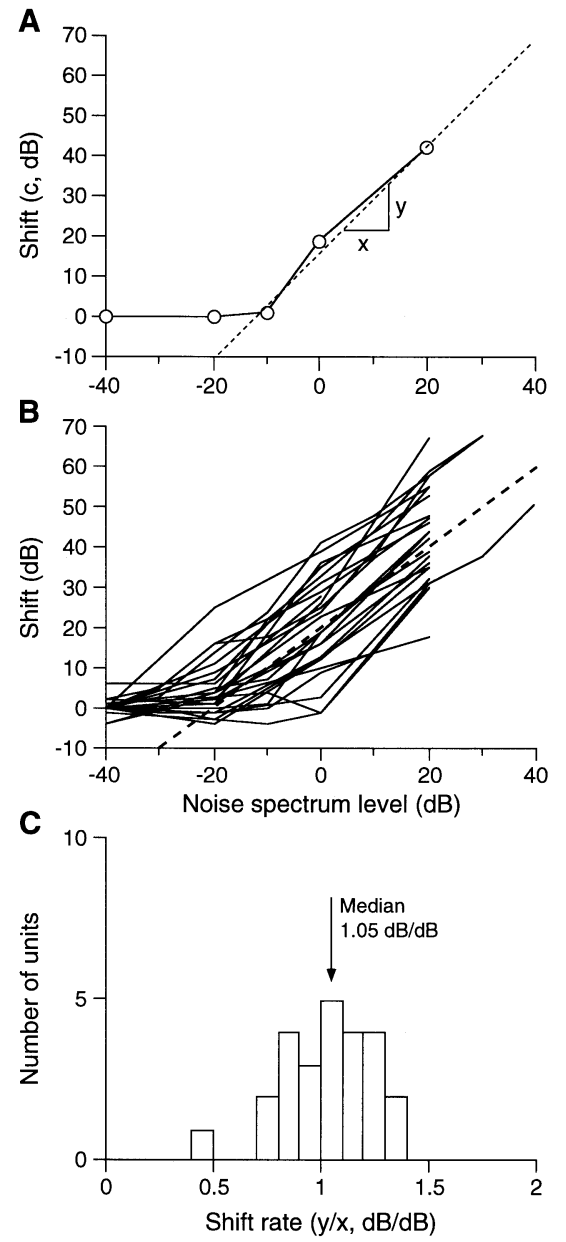

FIG. 3. N oise-induced shifts in the tone detection thresholds of type I units. A. Threshold shift is plotted as a function of noise spectrum level for the representative type I unit (unit number 7.10, BF $=36.3$ $\mathrm{kHz}$, experiment date: 08/25/1997). The unit's shift rate was derived by calculating the slope of a linear fit to the shift function (dashed line). B. Shift functions for all type I units in the present study. The dashed line indicates unity slope (i.e., $1 \mathrm{~dB}$ threshold shift per $1 \mathrm{~dB}$ increase in noise spectrum level); the line has been arbitrarily placed to correspond to linear increases in the shift functions of most units. C. Histogram showing the shift rates of all type I units. Arrow indicates the median shift rate of the type I distribution.

measured from all type I units in the present study. The dashed line shows a unity slope which represents the rate of threshold shift that is necessary to preserve the dynamic range of the neural representation (Gibson et al. 1985). The distribution of shift rates derived from linear fits to these data is plotted in Figure 3C. Type I units exhibited a median threshold shift of $1.05 \mathrm{~dB}$ for each 1-dB increase in background noise; consequently, this unit type is expected to maintain sensitivity to changes in signal level in high levels of background noise.

Figure 4 illustrates the compression effects of background noise on rate-level functions of type I units. These results are presented in terms of a scale factor, 


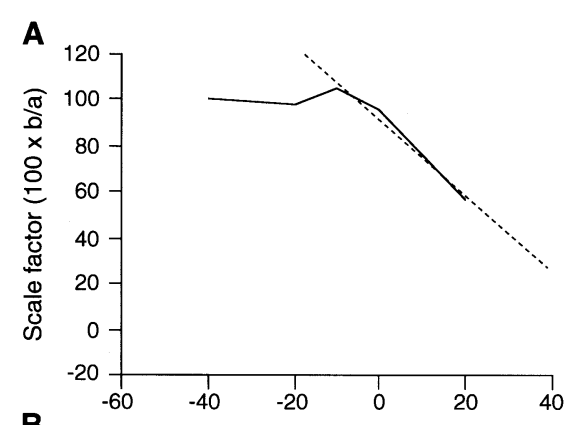

B
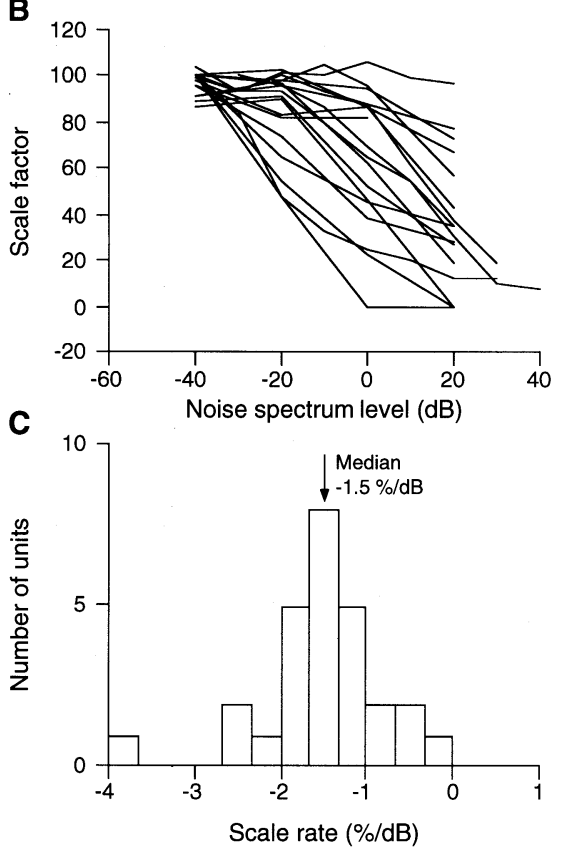

FIG. 4. Noise-induced compression of the tone-driven discharge rates of type I units. The dynamic range of rate responses is presented in terms of scale factors (see text for a detailed description of this measure). A. Effects of noise spectrum level on scale factors of the representative type I unit (unit number $7.10, \mathrm{BF}=36.3 \mathrm{kHz}$, experiment date: 08/25/1997). Scale factor rate (the rate of dynamic range compression) was derived from the slope of a linear fit to the transitional component of the scale factor function (dashed line). B. Scale factor functions for all type I units in the present sample. C. Distribution of scale factor rates for all type I units.

the ratio of the maximum tone-driven rate in background noise versus quiet conditions ( $100 \times b / a$, as illustrated by arrows in Fig. 2B). Scale factors of the representative type I unit are shown in Figure 4A. As with noise-induced threshold shifts, only noise spectrum levels above $0 \mathrm{~dB}$ produced major compression effects for this unit. These dynamic range changes were also approximately linear (dashed line). Figure $4 \mathrm{~B}$ plots the scale factor functions of all type I units in the present study. The threshold for noise compression differed widely within this sample, with most units showing scale factors greater than $50 \%$ at noise spectrum levels approaching $20 \mathrm{~dB}$. The few units that were sampled at higher noise spectrum levels also maintained some range of tone-driven activity (i.e., scale factors $>0 \%)$. Figure $4 \mathrm{C}$ shows the distribution of compression ratesfor type I units; these values represent the slopes of linear fits to the scale factor functions in Figure 4B. A median scale factor of $1.5 \%$ per $\mathrm{dB}$ suggests that most type I units lose their ability to encode tones in terms of discharge rate when noise levels reach 60-70 dB above the noise threshold.

\section{Effects of background noise on rate-level} functions of type $V$ and $O$ units

Figures 5 and 6 show the effects of background noise on the tone-driven responses of ICC type $V$ and $O$ units. Rate-level functions of a representative type $\mathrm{V}$ unit and its corresponding $d^{\prime}$ statistics are shown in Figure $5 A, B$. These data were obtained under quiet testing conditions and in two levels of background noise. In general, the effects of noise on the rate responses of type $V$ units were similar to those described previously for type I units; that is, tone detection thresholds shifted to higher levels and discharge rates compressed as the level of background noise increased. Decreases in d' suggest that noise-masking effects diminish the neural discrimination of changes in tone level. Figure $5 \mathrm{C}$ plots shifts in detection threshold as a function of noise spectrum level for each of the four type $V$ units that were recorded in the current study. This small sample reflects the fact that our sampling methods isolate type $V$ units much less frequently than other unit types. Two type $V$ units produced shift rates that approached a unity slope across several levels of background noise (dashed line), while the remaining two units displayed clear threshold shifts only at the highest noise spectrum level. Figure 5D plots the distribution of shift rates for the four type $V$ units. The median shift rate of this sample $(0.84 \mathrm{~dB} /$ $\mathrm{dB}$ ) impliesa slight decrease in the magnitude of noiseinduced threshold shifts relative to type I units, but such interpretations require a larger sample of type $V$ units than in the present study.

Type 0 units are dominated by inhibitory effects that drive stimulus-driven discharge rates to zero once sound levels reach 20-30 dB above threshold (Ramachandran et al. 1999). When type 0 units are tested with tones-in-noise, this powerful inhibition creates a rate-level function that is distinct from the largely excitatory responses of type $V$ and type I units. Ratelevel functions and their corresponding $d^{\prime}$ functions of a representative type 0 unit are shown in Figure $5 \mathrm{E}, \mathrm{F}$. Note that this unit exhibited a narrow range of excitatory responses under quiet conditions $\left(d^{\prime}>1\right)$ but showed strictly inhibitory responses in background noise $\left(d^{\prime}<-1\right)$. The threshold of the unit'sinhibitory responses was subject to the same noise-induced dynamic range shifts as the excitatory responses of type I and type $V$ units. The inhibitory threshold of 

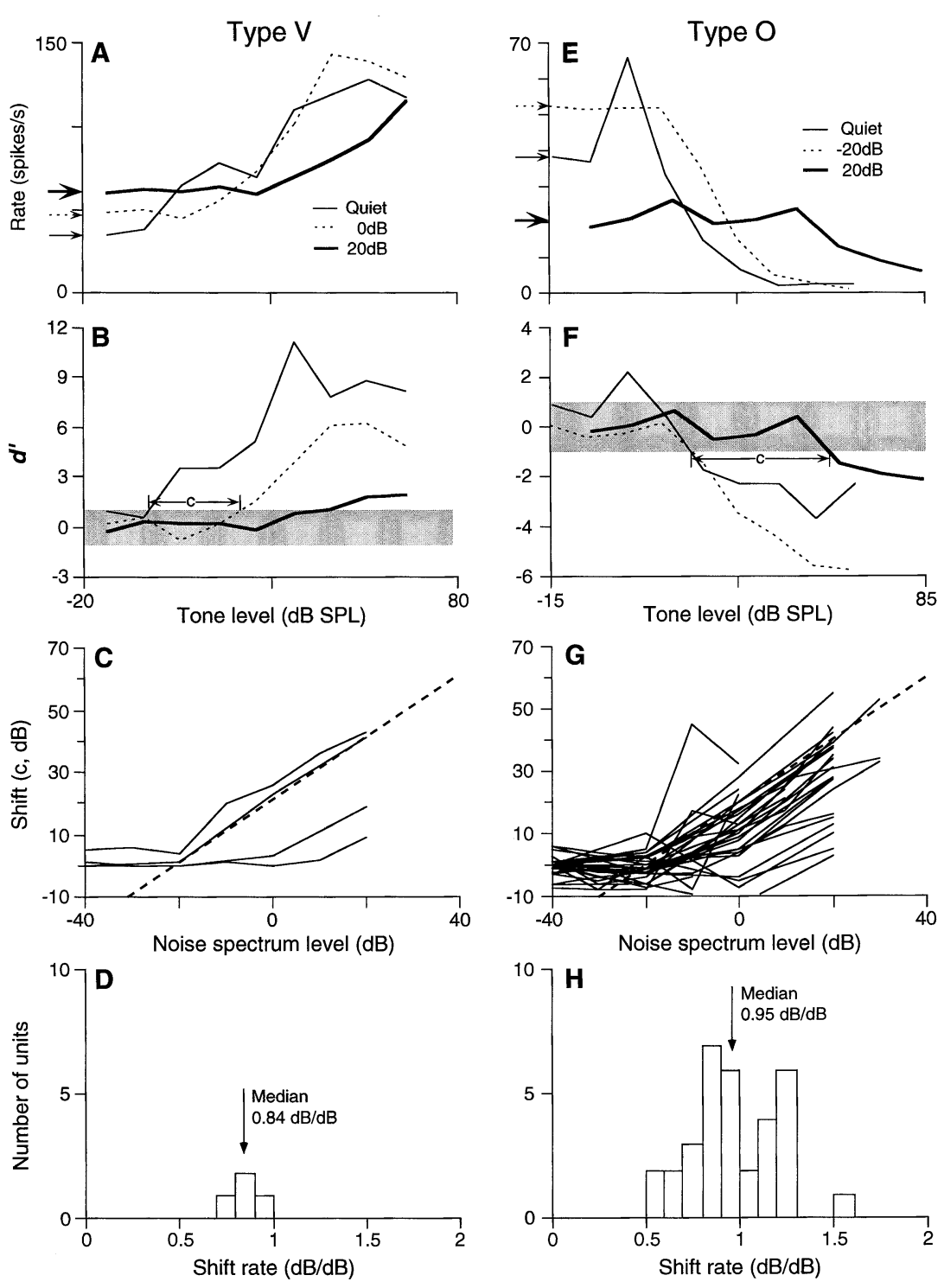

FIG. 5. Comparison of noise-induced threshold shifts for type $V$ (left panels) and 0 unit (right panels). A. Average rate-level curves for a representative type $\mathrm{V}$ unit (unit 3.03, $\mathrm{BF}=2.3 \mathrm{kHz}$, experiment date: 08/02/1999). Arrows indicate the background rates of each stimulus condition. B. $d^{\prime}$ functions based on the discharge rates shown in $\mathbf{A}$. Tone levels that failed to elicit detectable rate responses fall within the gray-shaded region. C. Threshold shift functions for all type $V$ units. Dashed lines have unity slopes. D. The distribution of shift rates for all type $\mathrm{V}$ units. E, F. Average rate-level curves and $d^{\prime}$ functions for a representative type 0 unit (unit 1.17, BF $=16.2$ $\mathrm{kHz}$, experiment date: 09/17/1997). G, H. Threshold shift functions and distributions for all type 0 units. type $O$ units was defined as the tone presentation level that evoked a rate decrease relative to the BR with a $d^{\prime}$ value of -1 . Threshold shifts were calculated in terms of differences between inhibitory thresholds in background noise versus quiet ( as shown by arrows labeled $\mathrm{C}$ in Fig. 5F). Shift magnitude is plotted as a function of noise spectrum level in Figure $5 G$ for all type 0 units in the present study. The distribution of slopes for these data is plotted in Figure $5 \mathrm{H}$. The median shift rate of type $O$ inhibitory thresholds was $0.95 \mathrm{~dB} /$ $\mathrm{dB}$ of background noise. Nonparametric statistical analysis found no significant differences between the shift rates of type I and type $O$ units (Mann-Whitney $U$ test, $p>0.2$ ).

Figure $6 \mathrm{~A}$ plots scale factors as a function of noise spectrum level for the representative type $V$ and $O$ units in Figure 5A,E. As for type I units, type $V$ units also exhibited substantial rate compression (i.e., small scale factors) with increasing levels of background noise. The distribution of scale factors for the four type $\mathrm{V}$ units in the present study produced a median value of $-1.65 \%$ per $d B$, as shown in Figure $6 B$.

Calculation of scale factors for type $O$ units is problematic because background noise exerts its major effects on this unit type by changing levels of background activity and not by tone-driven responses which tend to indicate complete inhibition at most stimulus levels above threshold regardless of the background stimulus condition. Relative to spontaneous discharge rates in quiet, the BR of the type 0 unit in Figure 5E increased in a noise spectrum level of $-20 \mathrm{~dB}$ (dashed line) because of excitatory responses to the background stimulus. By contrast, the BR decreased below spontaneous rates when this unit showed an inhibitory response to background noise with a spectrum level of $20 \mathrm{~dB}$ (bold line). As a result of the nonlinear changes in BRs and the constant inhibitory response at high BF tone levels, the unit's scale factors exhibited 

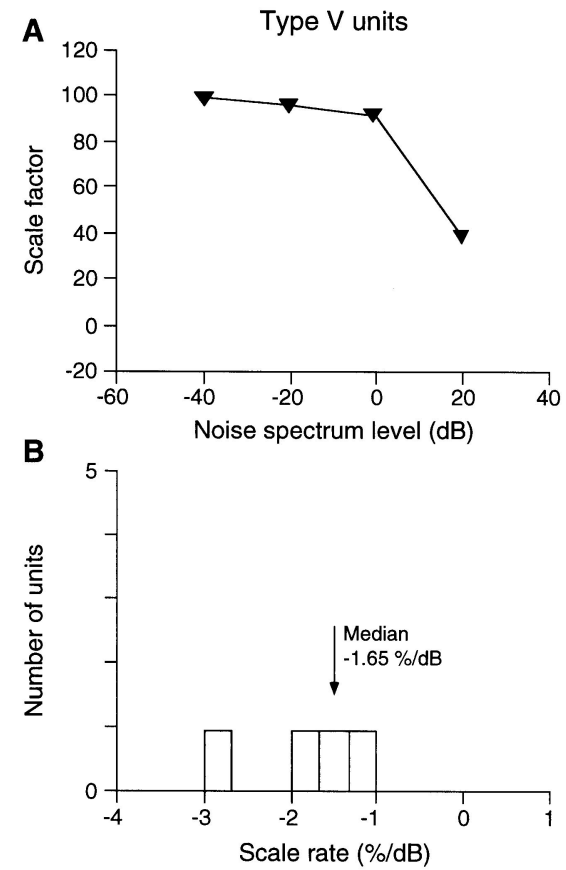
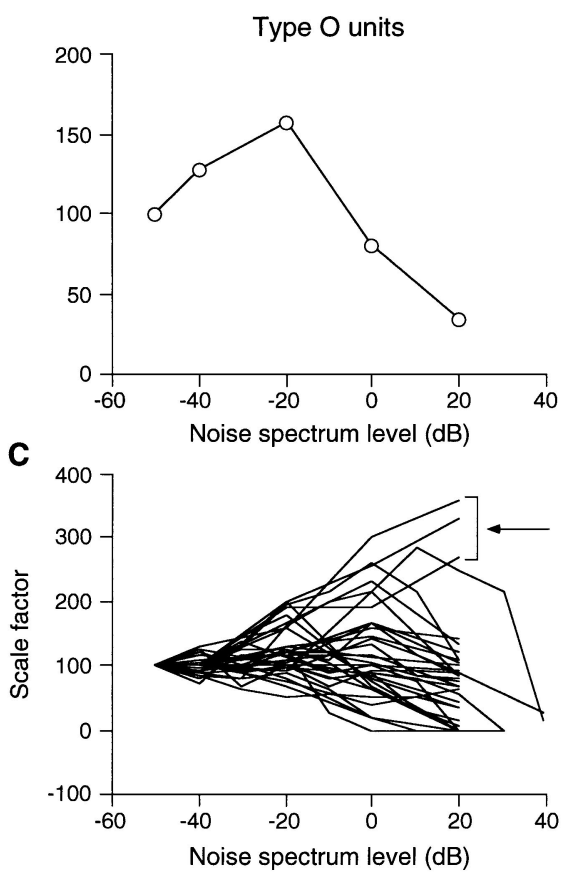

FIG . 6. Noise-induced rate compression for type $V$ and $O$ units. A. Scale factor functions for representative units (data are from the same units as in Fig. 5A, B). B. Histogram of scale rates for the four type $V$ units in the present sample. C. Scale factor functions for all type 0 units. Arrow points to three units that showed excitatory responses to high levels of background noise; the remaining units were inhibited by high levels of noise. first expansion and then compression with increasing levels of background noise. The scale factors of all type 0 units are shown in Figure 6C. A few units in this sample ( 3 out of 34) exhibited discharge rates to high levels of background noise that exceeded their maximum tone-driven rates. These excitatory effects produced the unusually large scale factors in Figure 6C (arrow).

\section{Effects of background noise on high-resolution} rate-level functions

The effects of background noise on the rate-level functions of auditory neurons have been investigated in many of the major nuclei of the ascending auditory pathway (ANF: Costalupes et al. 1984; Gibson et al. 1985; cochlear nucleus: Gibson et al. 1985; ICC: Rees and Palmer 1988; primary auditory cortex: Phillips and $\mathrm{H}$ all 1986). Most previous studies have measured the effects of noise on the dynamic range properties of auditory neurons by sampling single-unit responses with high-resolution (1-dB) changes in signal level. To facilitate quantitative comparisons with these independent observations, rate-level functions with a tone level resolution of $1 \mathrm{~dB}$ were obtained from a subset of units ( 83 units, 28 type I, 7 type V, and 48 type 0 units).

The effects of background noise on high-resolution rate-level functions were characterized by measuring rates of noise-induced dynamic range shifts and compression in several levels of continuous broadband noise. Prior to these calculations, $\mathrm{H}$ ill functions (Segel 1976) were fit to the data to smooth the transitional components of the rate-level functions. Actual physiological data and their corresponding curve fits are shown for the representative type $I$ and $O$ units in Figure 7A; these results were obtained in quiet and in continuous broadband noise with a spectrum level of $20 \mathrm{~dB}$. In accordance with previous conventions (e.g., Rees and Palmer 1988; May and Sachs 1992), shift magnitude was calculated as the difference between the tone levels that evoked the half-maximal excitatory response in noise versus quiet. Scale factors were calculated as the ratio of maximum tone-driven rates in noise versusquiet. As with the statistical measuresillustrated in Figure $5 \mathrm{~F}$, the shifts of type $\mathrm{O}$ units were calculated as the difference in BF tone levels that evoked half-maximal inhibitory response in noise versus quiet. The noise compression of the discharge rates of type $O$ units was not characterized because of strong nonlinearities in the noise responses of this unit type. Neurons that were studied with both high-resolution and statistical sampling paradigms showed no significant differences in their rates of noise-induced shifts or scale factor compression (paired t test, $p>0.1$ ).

Figure 7B compares rates of dynamic range shift for some of the principal single-unit response types along the ascending auditory pathway. The average shift rate of ICC type I units (solid lines) serves as the reference for this comparison. Variation within the sample of type I unitsisplotted in terms of the standard deviation of the mean (dashed lines). Type I units (labeled 1) in the ventral subdivision of the cochlear nucleus (VCN) are characterized bya purely excitatory receptive field that resembles the frequency response 

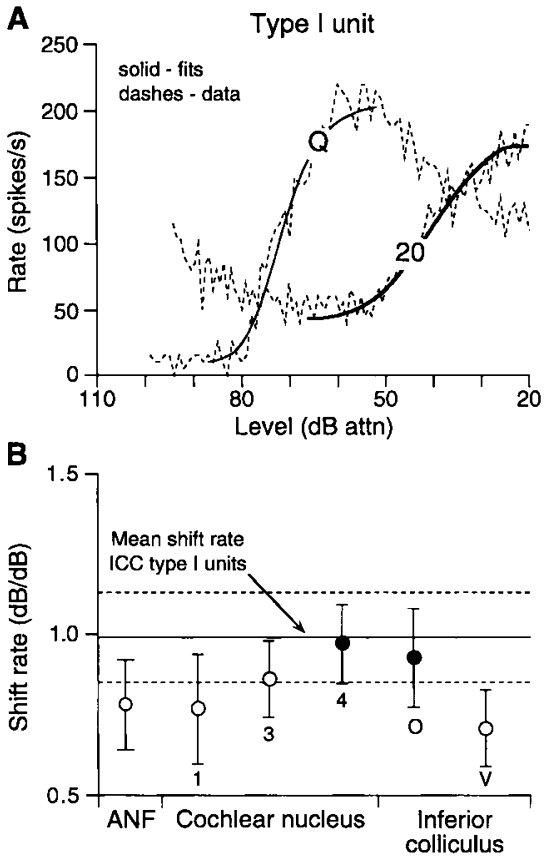

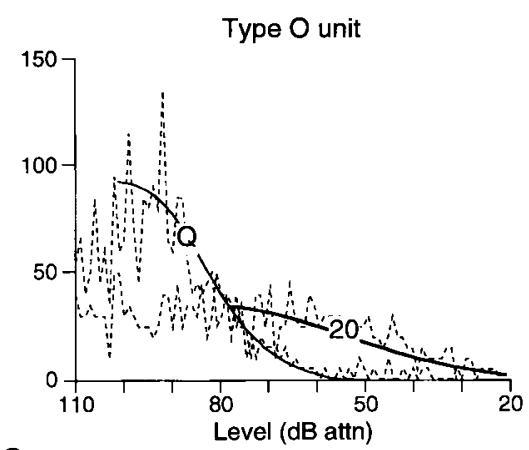

C

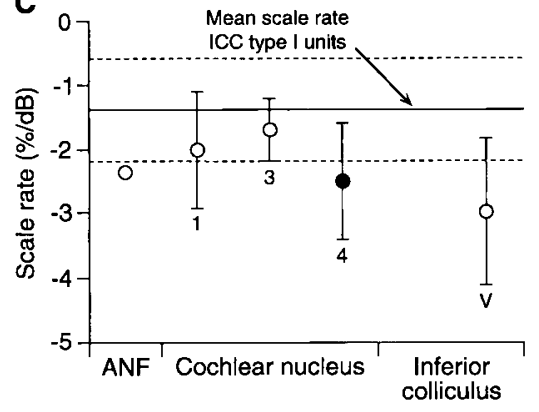

FIG. 7. Effects of background noise on the dynamic range of single-unit responses in the auditory nerve, cochlear nucleus, and inferior colliculus. A. Rate-level functions of two ICC neurons (type I unit 7.10, $\mathrm{BF}=36.3 \mathrm{kHz}$, experiment date: $08 / 25 / 1997$ and type 0 unit $1.17, \mathrm{BF}=16.2 \mathrm{kHz}$, experiment date: 09/17/1997). Hill functions were used to fit smoothed curves to the dynamic range component of actual physiological responses to tones in quiet ( $Q$ ) and in background noise (spectrum level $=20 \mathrm{~dB} S P L$ ). B. Comparison of noiseinduced threshold shifts for the major response types in the auditory nerve, cochlear nucleus, and ICC. The reference line is the mean shift rate of type I units; dashed lines indicate \pm 1 standard deviation (S.D.) of the mean. The mean ( \pm S.D.) of other unit types are plotted as symbols (error bars). O pen symbols identify unit types that were exclusively excited by BF tones; filled symbols mark unit types where shift rates were based on changes in inhibitory thresholds. C.Scale rates for units in the auditory nerve, cochlear nucleus, and ICC. The scale factors of DCN type IV units were calculated in terms of the strength of inhibitory responses. ICC type 0 units have been excluded from this analysis because of their nonlinear responses to background noise. N umerical labels in $\mathbf{B}$ and $\mathbf{C}$ are used in place of the standard cochlear nucleus nomenclature for type I, III, and IV units to avoid confusion with our non-numerical classification system for ICC units. maps of auditory nerve fibers. VCN type III units (labeled 3) exhibit lateral inhibitory sidebands that resemble ICC type I units and may serve as a source of input for these higher-order neurons (Ramachandran et al. 1999). Type IV (4) units are the projection neurons that link the dorsal cochlear nucleus (DCN) to the midbrain. These ascending inputs may play an important role in the formation of type 0 responses in the ICC (Ramachandran et al. 1999). Additional details of cochlear nucleus response properties can be found in Young et al. (1988). For unit types in which shift rates are based on excitatory responses (open symbols), mean shift rates ( $\pm S D$ ) in the auditory nerve, cochlear nucleus, and inferior colliculus were smaller than those exhibited by type I units. U nit types with strong inhibitory responses in the cochlear nucleusand inferior colliculus ( filled symbols) showed shift rates for inhibitory thresholds that were approximately equal to those of type I units.

Figure 7C compares noise compression effects in the auditory nerve, cochlear nucleus, and inferior colliculus. On average, ICC type I units exhibited less dynamic range compression (i.e., larger scale factors) than any other unit type. As the neural code for a tone-in-noise ascends from the auditory nerve to the cochlear nucleus and on to type I units in the inferior colliculus, units with excitatory response patterns (open symbols) show a progressive enhancement of dynamic range adjustments that help preserve the range of discharge rates that is available for stimulus representation in the presence of background noise. The small sample size of type $V$ units contributes to the large variability of scale rates for this unit type.

\section{Effects of background noise on tone detectability}

Signal detection measures were used to evaluate the effects of noise-induced dynamic range shifts and rate compression on the statistical magnitude of signal representations. $d$ ' values were calculated for tone-driven discharge rates as a function of both changes in absolute signal levels and changes in signal-to-noise ratios $(\mathrm{S} / \mathrm{Ns})$. The resulting distributions of $d^{\prime}$ values for ICC type I units are presented as statistical box plots in Figure 8. Figure 8A illustratesneural responsesto tones in four different noise levels ( -40 to $20 \mathrm{~dB}$ ) when the level of the test tone washeld $10 \mathrm{~dB}$ above the detection threshold for each background noise condition (i.e., constant S/ N). Changes in noise spectrum level produced no significant differences in the neural detection of the tone as long as this relative $10-d B S / N$ was maintained ( $p>0.1$, non parametric Friedman test). By contrast, the box plots in Figure 8B compare distribution of $d^{\prime}$ for a fixed absolute tone level of $40 \mathrm{~dB}$ SPL in the different levels of background noise. As $\mathrm{S} / \mathrm{N}$ s decreased at higher noise spectrum levels, lower 

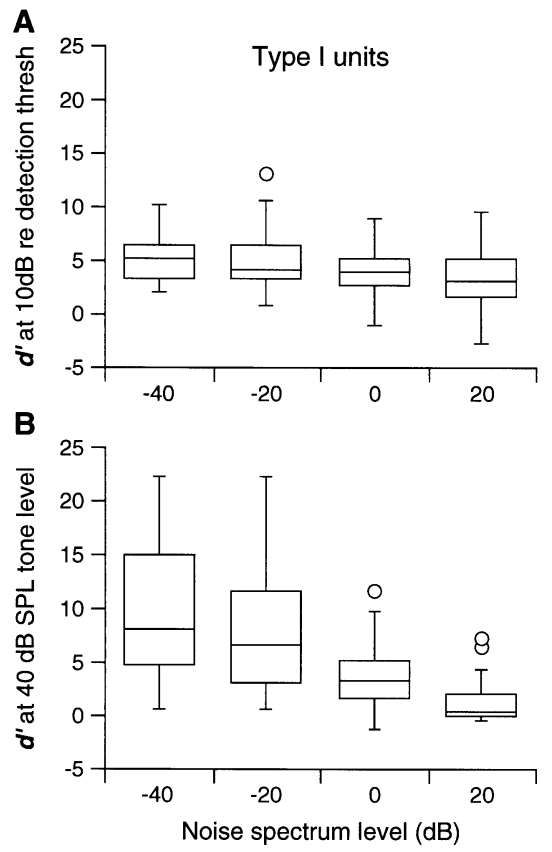

FIG. 8. Signal detection measures for the effects of noise on the tone-driven rates of type I units. A. d' scores for responses to a 10-dB re threshold tone in four noise spectrum levels. For this comparison, absolute tone levels vary across noise conditions to maintain a constant 10-dB ratio above the tone-in-noise detection threshold. B. d' scores for a 40-dB SPL tone in the same four noise spectrum levels. The upper and lower limits of each box plot indicate the interquartile range (middle $50 \%$ ) of the distribution of $d^{\prime}$ scores. Each box is divided at the median of the distribution. Error bars point to the most extreme data points within $\pm 1.5 \times$ the interquartile range. 0 utliers beyond this range are plotted as individual symbols.

$d^{\prime}$ scores indicate that the tone was masked more effectively. This loss of sensitivity was statistically significant $(p<0.01$, Friedman test).

Figure 9 shows median tone-detection d' scores as a function of noise spectrum level for each ICC unit type. These data are based on tone presentation levels of 10,40 , and $70 \mathrm{~dB}$ SPL. Although type I units produced the highest $d$ ' scoresfor almost all combinations of tone and noise, the tone detection capabilities of type I units were usually matched by those of type $V$ units except for the lowest levels of noise and the highest level of tone ( $70 \mathrm{~dB}$ SPL). Type 0 units consistently produced the lowest $d^{\prime}$ scores. This unit type showed positive $d^{\prime}$ scores in Figure 9A because most of the units in the sample were excited by 10-dB SPL tones; conversely, the $d^{\prime}$ scores of type $O$ units were negative in Figure 9B,C because higher tone levels evoked inhibitory responses. Responses where discharge rates are presumed inadequate to convey the presence of tones ( $d$ ' scores between \pm 1 ) are indicated by the gray-shaded region. All unit types showed a deterioration in the quality of the tone representation at higher noise spectrum levels, but the detection scores of type I and V units remained above threshold
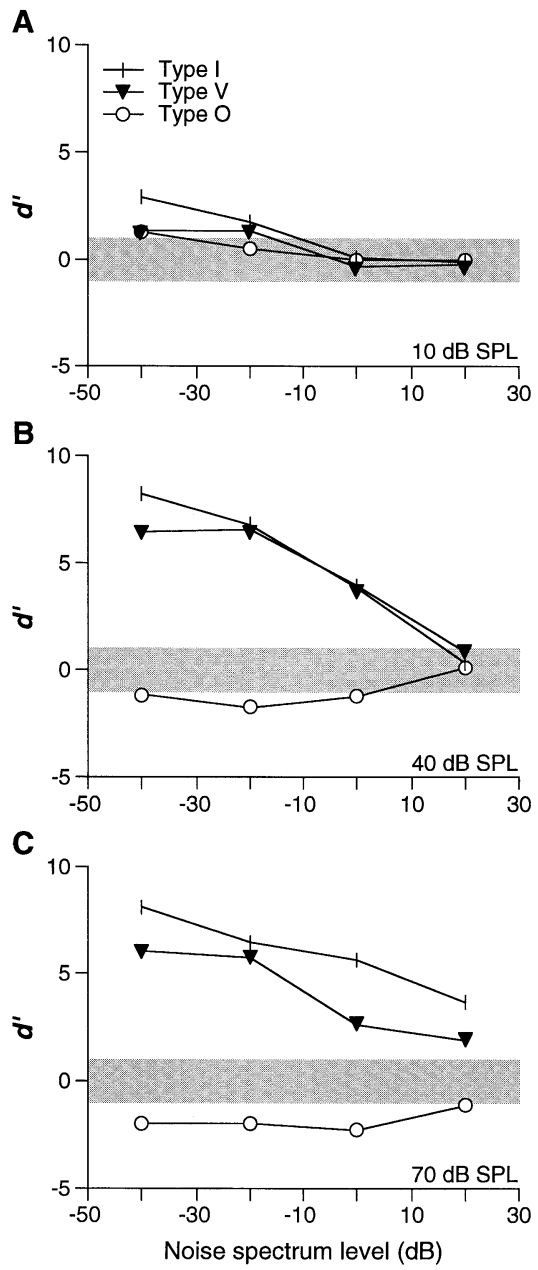

FIG. 9. A comparison of tone detection in noise across ICC unit types. A. Median d' scores for responses to a 10-dB SPL tone in four noise spectrum levels. B, C. d' scores obtained with tone levels of 40 and $70 \mathrm{~dB}$ SPL. Responses within the gray-shaded regions failed to detect the presence of the tone according to threshold criteria.

(outside the gray shaded regions) as long as tone levels exceeded noise spectrum levels by 10-15 dB.

Binaural influences on signal detection in background noise

Each of the three principal ICC unit types shows a different response pattern for binaural stimulation (Daviset al. 1999) and, therefore, may exhibit a unique sensitivity to tones in noise under more natural freefield conditions where acoustic filtering effects of the head lead to interaural level disparities. Binaural influences on the detection of tones in noise were investigated by measuring rate-level functions for $\mathrm{BF}$ tones with a 20-dB contralaterally dominant interaural level difference (ILD) against backgrounds of three different noise ILDs: a 20-dB contralaterally dominant ILD, a $0-d B$ midfrontal ILD, and a-20-dB ipsilaterally dominant ILD. Figure 10A shows $d^{\prime}$ scores that were derived 

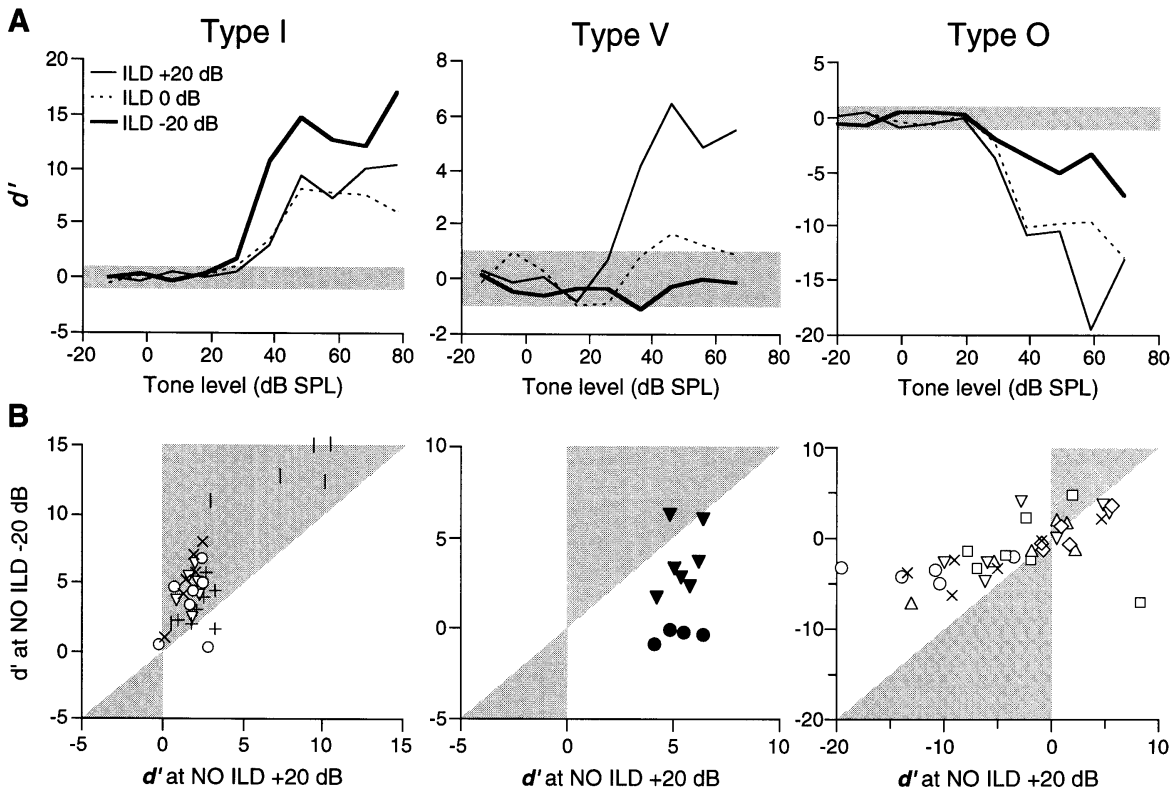

FIG. 10. Effects of ILD on the detection of tones in noise. A. d' functions for representative type I, type $\mathrm{V}$, and type $\mathrm{O}$ units. The noise level of the background noise was fixed at $10 \mathrm{~dB}$ re threshold (approximately $0-d B$ spectrum level) in the contralateral ear and varied in the ipsilateral ear to produce ILDs of $-20,0$, and $+20 \mathrm{~dB}$. Tone levels were changed simultaneously in the two ears to maintain a fixed ILD of $+20 \mathrm{~dB}$. Responses that failed to attain the detection threshold are indicated by the gray-shaded region. B. Scatter plot of $d^{\prime}$ scores for responses to the same tone levels in noise ILD s of +20 vs. $-20 \mathrm{~dB}$. The different symbols indicate values from different units. Responses within the gray-shaded regions showed enhanced tone detection at the $+20-d B$ noise ILD. from the rate-level functions of representative units for the three major response types. The type I unit showed an improvement in signal detection when the background noise was presented with a-20-dB ILD (bold lines). This stimulus condition simulates movement of the masker to ipsilateral locations remote from the signal. Psychoacoustic experiments (e.g., Saberi et al. 1991) have demonstrated a similar directionally dependent release from masking in human listeners. By contrast, the type $V$ and $O$ units exhibited threshold enhancements relative to ipsilateral masking effects when the simulated contralateral location of the signal was approached by background noise at midfrontal (dashed lines) or contralaterally dominant sound locations (thin lines). Spatial masking release is not predicted under these conditions by psychophysical results in free-field masking experiments.

Figure 10B presents results from additional units in our study of binaural interactions ( 6 type I, 2 type V, and 6 type $O$ units). Each symbol contrasts $d^{\prime}$ values that were obtained at the same BF tone level with background noise ILDs of +20 (abscissa) versus -20 $\mathrm{dB}$ ( ordinate). The ILD of the tone was maintained at $+20 \mathrm{~dB}$. Each unit in the sample was tested with several tone levels and therefore contributed multiple points to the plots. Shaded regions identify d' scores that indicate enhanced signal detection with the negative ILD background noise. Data falling within these regions replicate the psychoacoustic observation that auditory signals are more detectable when they are spatially separated from masking noise. Only type I units exhibited a consistent enhancement of tone representations under these conditions.

\section{DISCUSSION}

Results obtained with monaural stimuli suggest that ICC neurons in decerebrate cats manifest the same general patterns of noise-induced dynamic range shifts and compression that have been described in other principal nuclei of the auditory system. Within the ICC, type I units displayed the most sensitive average rate responses to narrowband signals in broadband masking noise. This enhanced representation showed further improvement when tests were performed with binaural stimuli that simulated free-field conditions with spatial separation between signal and masker.

M onaural effects of background noise on the discharge rates of ICC neurons

The effects of broadband noise on single-unit responses in ICC also have been measured in anesthetized guinea pigs (Rees and Palmer 1988). In place of the classification system used in the present study, neural responses were characterized in this earlier report on the basis of monotonic versus nonmonotonic rate-level functions for monaural tones. On average, single-unit responses in the ICC of guinea pigs showed noise-induced dynamic range shifts of $0.97 \mathrm{~dB}$ for each 1-dB increment in background noise regardless of the shape of rate-level functions. O ur own prior studies (Ramachandran et al. 1999) found that units with monotonic rate-level functions in decerebrate cats typically displayed type I response properties; whereas, most nonmonotonic units had type 0 characteristics. Consistent with previous findings in guinea pigs, the present study found no significant differences in the 
shift rates of type I (monotonic) and type $O$ (nonmonotonic) units.

Noise compression effects also did not differ significantly between ICC response types in anesthetized guinea pigs when single-unit responses were grouped on the basis of the shape of rate-level functions (Rees and Palmer 1988). Although statistical comparisons of scale factor rates were not performed for type I and type 0 units in the present study because of the nonlinear noise responses of type 0 units, qualitative comparisons suggest that the discharge rates of most ICC units in the two species compressed at approximately the same rate over the same range of noise spectrum levels (e.g., compare the scale factor functions in Figures 4B and $6 \mathrm{C}$ in this report with Figure 8 in Rees and Palmer 1988). O ne clear difference between the two studies, however, is the magnitude of inhibitory influences shown by the rate-level functions of type $O$ (nonmonotonic) units. In decerebrate cats, the discharge rates of the majority of type 0 units were effectively eliminated by high levels of tone or background noise ( Fig. $5 \mathrm{E})$. In anesthetized guinea pigs, nonmonotonic units displayed tone-driven rates that remained above spontaneous activity at high stimulus levels.

The diminished noise compression effects shown by nonmonotonic units in anesthetized guinea pigs may be explained by methodological factors that affected the strength of ICC inhibitory influences in the Rees and Palmer (1988) study relative to our present results in decerebrate cats. For example, the short tone bursts with rapid presentation rates used as stimuli by Rees and Pal mer primarilyevoke onset responses that tend to show less nonmonotonicity at high stimulus levels. These transient responses, therefore, are presumed to belesssensitive to level-dependent inhibitory effects (Rees 1992). The balance of excitatory and inhibitory interactions that shape ICC discharge rates may also be modified by the effects of anesthesia (Kuwada et al. 1989). Relative to decerebrate cats, barbiturate-anesthetized cats show less spontaneous activity and a stronger bias toward onset activity ( Ramachandran et al. 1999). Both of these changesin responsivity make it difficult to characterize the normal strength of inhibitory influences in the presence of anesthesia. 0 ur present studyavoided these confounds by characterizing noise effects in unanesthetized decerebrate cats.

0 ther investigators have measured masked thresholdsin the ICC of barbiturate-anesthetized cats to evaluate neural mechanisms for sharpening auditory frequency resolution within the central ner vous system (Ehret and Merzenich 1988). U sing methods devised in psychoacoustic studies (Fletcher 1940), critical ratios were computed in these studies as the difference between the masked detection threshold for monaural tones and the spectrum level of masking noise. The rationale for this procedure is that neurons with sharp frequency tuning will produce lower tone detection thresholds in broadband noise (i.e., smaller critical ratios) because a reduction in the effective bandwidth of the masker improves the signal-to-noise ratio of sound energy within a neuron's receptive field. Ehret and $M$ erzenich found that low critical ratiosare characteristic of ICC units with strong lateral inhibition. Our prior studies in anesthetized cats have indicated that units with lateral inhibition (level tolerant units) display the same basic response properties as type I units in decerebrate cats (Ramachandran et al. 1999). From this perspective, the present observation that type I units provided the most sensitive representation of monaural tones in noise supports Ehret and Merzenich's earlier findings that lateral inhibition improves signal encoding in background noise. Rhode and Greenberg (1994) reached similar conclusionsin their study of the cochlear nucleus.

\section{Binaural processing of auditory signals in background noise}

Two basic stimulus paradigms have been used to investigate the effects of binaural interactions on the detection of auditory signals in background noise. In one procedure, natural binaural effects are produced by presenting signals and maskers in free field; in the other, virtual sound fields are created by closed-field simulation of the spatial cues that arise from headrelated transfer functions (HRTFs: the filter functions describing the transformation of free-field sounds to energy at the tympanic membrane).

Previous free-field studies have shown that singleunit responses in the inferior colliculus are sensitive to variations in stimulus location (Aitkin et al. 1984). The spatial receptive fields of ICC neurons can arise from simple transformations of BF sound-pressure levels that are produced by the directional filtering properties of the pinna (Phillips et al. 1982), or from more complex binaural interactions that are determined by the relative amplitude and timing of acoustic stimuli at the two ears (Delgutte et al. 1999). Correlations between free-field effects on neural responses to tones in noise and the psychoacoustic phenomenon of masking release have been investigated in a previous electrophysiological study of paralyzed frogs (Ratnam and Feng 1998). Single-unit responses in the amphibian ICC were distinguished on the basis of transient (phasic) or sustained (tonic) responses to tonal stimuli. Tone detection thresholds improved when transient units were tested with spatially separated tones and noise, but a loss of sensitivity was observed for tonic units under the same stimulus conditions. Although the receptive field properties of neurons in the amphibian ICC have not been classified, type 0 units 
in decerebrate cats show transient onset responses at stimulus levels above the circumscribed range of their excitatory response area; type I units have sustained responses. If our present classification system can be extended to the frog, the results of Ratnam and Feng suggest that type $O$ units provided an enhanced detectability of tones in noise relative to type I units. The physiological basis of this apparent contradiction to results in Figure 10 remains to be determined with temporal manipulations of binaural stimuli. O nly ILDbased binaural interactions were investigated in the present study. The role of ILD cues in the sound localization behaviors of the frog islikely to be of secondary importance to binaural temporal interactions because of the predominance of low-frequency hearing in the species and the existence of an internal acoustic coupling between the two ears that may attenuate interaural level differences (Narins et al. 1988).

The majority of psychoacoustic and physiological studies of spatial masking release have used close-field procedures to allow precise independent manipulation of ILD, interaural time difference (ITD), and spectral information at the two ears. The importance of ITD cues on signal detection has been known since the early psychoacoustic observation that a simple inversion of the interaural phase relationship between a tone and background noise could decrease the tone detection threshold by as much $15 \mathrm{~dB}$ (see M cFadden 1975 for a review of human psychoacoustic literature). Physiological correlates of the temporal factors that govern binaural masking level differences (BMLDs) have been identified for low-frequency neurons in the inferior colliculus of anesthetized guinea pigs ( Caird et al. 1991; Jiang et al. 1997). Although temporal masking effects have not been characterized in the ICC of decerebrate cats, type $V$ units are presumed to receive ascending inputs from ITD-sensitive neurons in the MSO and provide the most sensitive representation of ITD information of the major ICC response types (Ramachandran and May 1999a).

Detailed acoustic analyses of the HRTF of the cat (Musicant et al. 1990; Rice et al. 1992) have provided accurate assessments of interaural differences of level, time, and spectral shape that result from directional changes in free-field sound sources. Virtual space stimuli can be simulated from these measures to determine how binaural acoustic interactions influence the detection of free-field sounds in background noise. Recent studies in the inferior colliculus of anesthetized cats (Lane et al. 1999) have used HRTF-based stimuli to show that the directionally dependent masking of auditory signals in background noise can result from excitatory (line busy) effects related either to responses evoked by the noise masker or to suppressive effects mediated through inhibitoryinfluences in the absence of noise-driven activity. O ur previous studies (Davis et al. 1999) have found that contralateral noise produces strong excitatory responses in both type I and type $\mathrm{V}$ units; therefore, the loss of signal representation that was evident for these units under monaural stimulus conditions presumably was due to excitatory masking effects (Fig. 9). In the case of type I units, excitatory response areas are decreased in bandwidth at high stimulus levels by inhibitory in puts (Ramachandran et al. 1999). Broadband maskers, therefore, are likely to exert their influences on this unit type through a combination of excitatory and suppressive effects. It is interesting to note that type I units exhibit an enhanced signal representation relative to type $V$ units at high stimulus levels (Fig. 9C). This observation suggests that monaural inhibitory mechanism are capable of attenuating the masking of narrowband signals by broadband noise even when spatial factors are not favorable for binaural masking release.

Type $V$ units are excited by ipsilateral presentations of broadband noise. Type I units, on the other hand, are inhibited by ipsilateral noise and show an additional narrowing of the excitatory receptive field for contralateral stimuli (Davis et al. 1999). These differences in ipsilateral response properties suggest that excitatory versus suppressive masking effects dominate the responses of the two unit types for signals and maskers that arrive at the two ears from sound sources in opposite sound fields. When the present study simulated these free-field conditions by manipulating the ILD of binaural stimuli, only type I units displayed spatial masking release for contralateral signals when the masker was shifted to an ipsilateral location (Fig. 10).

\section{Comparisons of tone-in-noise representations across levels of processing}

Figure $11 \mathrm{~A}$ illustrates the putative ascending path ways for the ICC response types that were investigated in the present study. Based on similarities of monaural and binaural response properties (Ramachandran et al. 1999; Davis et al. 1999; Ramachandran and May 1999a,b) and the effects of pharmacological manipulations (Davis et al. 1999), type I units appear to derive their dominant ascending inputs from the ventral cochlear nucleus (VCN) via the lateral superior olive (LSO). Inferences about transformations in the neural information that passes directly from the LSO to the inferior colliculus are complicated by a lack of quantitative data on the encoding of tones in noise by LSO neurons; however, it is possible to speculate on how the quality of discharge rate representations from the cochlear nucleus may be improved by the combined effects of noise-cancellation circuitry in the LSO and ICC.

Cochlear nucleus sperical and globular bushy cells 
A

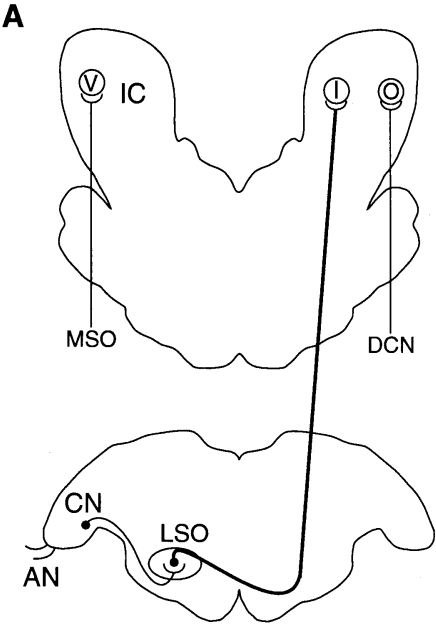

B

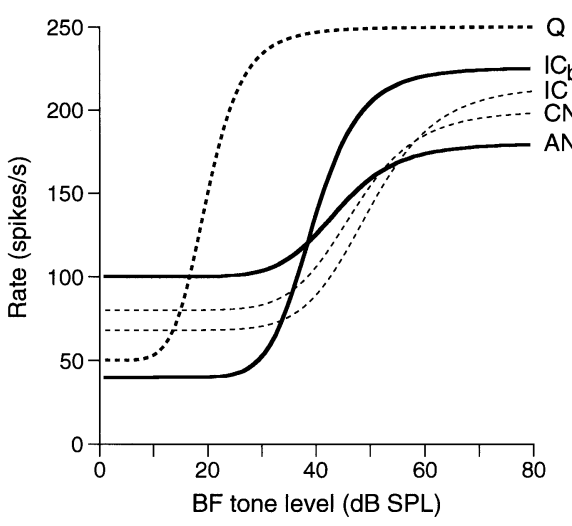

FIG. 11. Ascending pathways for the representation of tones in noise. A. Putative brainstem projections to the major ICC response types. B. Idealized rate-level functions illustrating transformations in the dynamic range of the neural representation of tones in noise as rate information ascends from the auditory nerve, to cochlear nucleus, and inferior colliculus. Auditory nerve responses are shown for tones in quiet $(\mathrm{Q})$ and in background noise with a 30-dB spectrum level (AN). Cochlear nucleus responses $(\mathrm{CN})$ are based on the median discharge rates of type I (1) units, which have been proposed as a source of input for ICC type I units. Inferior colliculus responses reflect the median discharge rates of type I units during testing with monaural (IC) and binaural stimuli $\left(\mathrm{IC}_{\mathrm{b}}\right)$. Binaural masking release was maximized by setting the ILDs of tones and noise to +20 and $-20 \mathrm{~dB}$, respectively. link primaryafferent (auditory nerve) inputs from the cochlea to the LSO, medical nucleus of the trapezoid body (MNTB), and medial superior olive MSO (see Cant 1992 for a review). Along this pathway, powerful endbulb synapses create a precisely timed coupling of auditory nerve inputs to the cochlear nucleus (Ryugo and Fekete 1982; Ryugo and Sento 1991) that allows bushy cells to be identified in electrophysiological studies by their primary-like response properties (Rhode et al. 1983). Primary-like units in the cochlear nucleus exhibit a V-shaped excitatory receptive field that is similar to the frequency response map of the ICC type $V$ unit (Fig. 1). This receptive field organization is designated a type I (numerical 1 ) response in physiological studies of the cochlear nucleus (Shofner and Young 1985).

Figure 11B illustrates how the discharge rate encoding of signals in noise is transformed as the neural representation passes from the auditory nerve to the cochlear nucleus type I unit (this specification refers to the Roman numeral 1) and then the ICC type I unit (this specification refers to the unit's i-shaped receptive field). These idealized BF tone rate-level functions were created by calculating the median noise-induced threshold shift and rate compression for populations of neurons in decerebrate cats. The electrophysiological data for each tone-in-noise function were recorded in the presence of a $30-d B$ re threshold noise spectrum level. Auditory nerve and cochlear nucleus measures were calculated from archival data of Gibson et al. (1985). As a reference for gauging the magnitude of noise effects, the auditory nerve rate-level function for testing in quiet is shown (bold dashed line). Relative to auditory nerve responses in background noise (bold solid line), the rate-level function of VCN type I (1) units shows only slightly more shift and less rate compression. Statistical tests of differences in the dynamic range adjustments of auditory nerve fibers and VCN neurons did not attain significance in the Gibson et al. study. These similarities led Gibson and colleagues to conclude that background noise indirectly influences VCN discharge rates through the effects of two-tone suppression and short-term adaptation that occur in the cochlea (Costalupes et al. 1984).

The rate-level functions for type I units in Figure 11B suggest that noise-cancellation effects within the ICC have enhanced the neural encoding of signals in noise. In addition to decreases in rate compression that are noted under both monaural and binaural stimulus conditions, an improvement in sensitivity is gained by binaural interactions that can decrease the tone detection thresholds of type I units by as much as $15 \mathrm{~dB}$. As discussed above, this masking release is presumed to result from ipsilateral inhibitory mechanisms that suppress excitatory responses to contralateral noise stimuli. The binaural rate-level function of the type I unit reveals this decrease in noise-driven activity as a drop in background rates at subthreshold tone levels (i.e., the leftmost data points on the ratelevel functions).

It is not known to what extent auditory processing in the LSO contributed to the dynamic range adjustments of the ICC type I rate-level functions in Figure 11B, but LSO neurons do show features of the inhibitory interactions that allow type I units to enhance the neural encoding of signals in noise. Under monaural stimulus conditions, the frequency response maps of LSO principal cells exhibit lateral inhibitory sidebands that constrain the excitatory bandwidths of their receptive fields (Caird and Klinke 1983). LSO neurons also receive contralateral inhibitory in puts from globular bushy cells via the MNTB (Morest 1968). Although the projection of the cochlear nucleus into the MNTB is sharply tuned and shows a precise tonotopic organization, MNTB inputs to the LSO are highly divergent 
(Gabriele and Henkel 1997). This innervation pattern increases the frequency bandwidth of inhibitory responses to contralateral stimuli. The broadband inhibitory influences that LSO neurons display under both ipsilateral and contralateral stimulus conditions suggest that precursors of the noise-cancellation circuitry of type I units exist at lower levels of processing in the auditory brainstem.

A subset of type I units may be created by ascending projections from stellate cells in the VCN that reach the ICC without the synaptic intervention of the superior olivary nuclei (see Cant 1992; Oliver and Huerta 1992 for review). These units respond to acoustic stimulation with a regularly timed "chopping" discharge rate (Rhode et al. 1983) and produce either a type I (1) frequency response map or a type III (3) map that is characterized by a central region of excitation and lateral inhibitory areas (Shofner and Young 1985). If VCN type I (1) units were to provide the dominant excitatory inputs to some ICC type I units, then convergence with a suitable inhibitory input would be necessary to create the lateral inhibitory areas. This possibility is supported by findings that iontophoresis of bicuculline in ICC can sometimes expand the narrow excitatory area in a type I-like map into the Vshaped response area of a type V-like map (Yang et al. 1992; LeBeau et al. 1995). Similarities in the receptive field organization of VCN type III (3) units and ICC type I units suggest that direct VCN inputs might also dictate some characteristics of the ICC type I response pattern. This interpretation is supported by the observation that a minority of ICC type I units are insensitive to binaural temporal cues, as would be expected for higher-order neurons receiving inputs from the strongly binaural LSO (Ramachandran and May 1999a,b). Unlike type I units, VCN type I (1) and type III (3) units exhibit broadly tuned excitatory response areas at high stimulus levels (Young et al. 1988) and weak inhibitory inputs from the opposite ear (Pfalz 1962). In addition, the noise-induced shifts and rate compression of type I (1) and type III (3) units tend to be smaller than ICC type I units (Fig. 7). If VCN type I (1) or type III (3) projections to the ICC do contribute to type I responses, these differences in response characteristics suggest that the signal-in-noise processing capabilities of type I units are further modified by alternate sources of ascending binaural inputs like the LSO and local inhibitory influences within the ICC.

Type $V$ units in the ICC are thought to receive their dominant afferent inputs from the MSO (Ramachandran et al. 1999; Davis et al. 1999). The effects of background noise on auditory signal processing in this nucleus have not been studied in detail. Like LSO neurons, MSO principal cells receive their dominant ipsilateral inputsfrom spherical bushycells in the VCN (see Cant 1992; Schwartz 1992 for a review). Whereas the LSO to type I projections appear to modify these cochlear nucleus inputs with binaural inhibitory interactions that enhance the encoding of ILD cues, processing specializations in the MSO to type $\mathrm{V}$ pathway impart a sensitivity to the low-frequency temporal properties of binaural stimuli (Ramachandran and May 1999a). A potential consequence of this temporal specialization is the observation that type $V$ units invariably have BFs that fall within the range where most peripheral auditory neurons exhibit good phaselocked responses $(<3 \mathrm{kHz})$. In addition to the constraints on frequency encoding that are imposed by these frequency limitations, further questions concerning a primary role of type $V$ units in the auditory representation of signals in noise are raised by results shown in Figure $9 \mathrm{C}$, which indicate that the more broadly tuned excitatory receptive fields of the type $V$ units produce a less sensitive representation at high stimulus levels relative to the more sharply tuned excitatory response areas of type I units. Under binaural conditions, type $V$ units displayed their largest spatial masking release when ILD manipulations simulated cospatial signals and maskers (Fig. 10B). In combination, these results cast doubt on the ability of the type $V$ pathway to support psychoacoustic performance in terms of discharge rate over a wide range of stimulus conditions; nevertheless, type $\mathrm{V}$ units must play an important role in temporal processes that contribute to binaural masking release for low-frequency stimuli (Jiang et al. 1997; Palmer et al. 1999) .

Type $O$ units in the ICC are thought to be derived from direct projections from fusiform and giant cells in the dorsal cochlear nucleus (DCN). In electrophysiological experiments these neurons exhibit type 0 like frequency response maps (Ramachandran et al. 1999) which are designated type IV (4) responses in studies of the cochlear nucleus (Young and and Brownell 1976). Additional evidence linking DCN type IV (4) inputs to ICC type $O$ units comes from the obser vation that some type $O$ responses are eliminated by surgical or pharmacological manipulations of the fiber tracts that convey the axons of DCN principal cells to the inferior colliculus (Davis 1999).

In prior studies of the effects of background noise on discharge rates in the cochlear nucleus (Gibson et al. 1985), type IV (4) units were the only response type to show significantly larger noise-induced dynamic range shifts than auditory nerve fibers (ANFs). These results suggest that the $D C N$ representation of signals in noise is determined not only by cochlear mechanisms but also by the action of inhibitory inputs from multiple sources within and beyond the cochlear nucleus (Lorente de No 1933; see Cant 1992 for review). Comparisons of these previous measures with our present findings suggest that the median shift rates 
of DCN type IV (4) units and ICC type 0 units are equivalent to that of type I units (Fig. 7C). Nevertheless, because type $O$ units show the same contradictory patterns of cospatial masking release as type $V$ units (Fig. 10B), their binaural responses appear inadequate to support psychoacoustic masking phenomenon.

Processing models that ascribe an important role for the DCN type IV (4) to ICC type 0 pathway in the monaural encoding of auditory signals also must take into account the strong nonmonotonicities that both unit types exhibit at high sound-pressure levels for narrowband stimuli (Ramachandran et al. 1999). Similar observations in auditory cortex (Brugge and Merzenich 1973; Phillips et al. 1985) have led to the proposal of a processing scheme in which the nonmonotonic rate-level function defines a "best intensity" for individual neurons in much the same manner as best frequencies are tonotopically mapped by populations of neurons with frequency-tuned responses. Based on results in thisstudy, a problem with thisputative distributed representation of sound-pressure level is that type 0 units in decerebrate cats do not exhibit enough threshold variability to encode the full range of signal levels that constitute normal auditory function (Ramachandran et al. 1999).

Previousstudies of the encoding of tones in noise by neuronsin the primaryauditorycortex of anesthetized cats have reported dynamic range shift rates of $1 \mathrm{~dB} /$ $\mathrm{dB}$, with verylittle compression of maximum discharge rates (Phillips and Cynader 1985; Phillips and Hall 1986). These results suggest that rate information conveyed by the ICC may be further enhanced as neural representation sof signalsin noise ascend to the cortex. Although the efferent projections that link auditory cortex to inferior colliculus are a potential source of added dynamic range adjustments, the influence of cortical feedback systems cannot be evaluated in the decerebrate preparation because these descending path ways are transected as a consequence of the surgical procedure. It is interesting to note that single units in the VCN of awake and behaving cats show less rate compression for tones in noise than units in decerebrate cats (May and Sachs 1992). This "decompression" of rate information is presumably achieved by olivocochlear feedback systemsthat reduce noise sensitivity in the auditory periphery (Winslow and Sachs 1987). Descending cortical influences also are potentially capable of improving neural encoding in noisy environments, perhaps by adapting the excitatory receptive field properties of ICC neurons to the bandwidth of auditory signals ( Gao and Suga 1998; Yan and Suga 1999). Direct tests of the functional significance of these cortical feedback systems await future studies in awake, intact animals.

\section{ACKN O W LED G MENTS}

E. Young contributed archival data that allowed comparisons of present results with noise effects in the auditory nerve and cochlear nucleus. E. Young, A. Rees, and J. Yu provided helpful comments during manuscript preparation. This research was supported by NIDCD grant 5 P60DC00979.

\section{REFEREN CES}

Aitkin LM, Gates GR, Phillips SC. Responses of neurons in the inferior colliculus to variations in sound source azimuth. J. Neurophysiol. 52:1-17, 1984.

Aitkin LM, Webster WR, Veale JL, Crosby DC. Inferior colliculus. I. Comparison of response properties of neurons in central, pericentral, and external nuclei of adult cat. J. Neurophysiol. 38:11951207, 1975.

BRUGGe JF, MERZENICH MM. Responses of neurons in the auditory cortex of the macaque monkey to monaural and binaural stimulation. J. Neurophysiol. 36:1138-1158, 1973.

CAIRD D, KLINKE R. Processing of binaural stimuli by cat superior olivary complex neurons. Exp. Brain Res. 52:385-399, 1983.

Caird D, Palmer AR, Rees A. Binaural masking level difference effects in single units of the guinea pig inferior colliculus. H ear. Res. 57:91-106, 1991.

CANT NB. The cochlear nucleus: Neuronal types and their synaptic organization. WeBster DB, Popper AN, FAY RN. The Mammalian Auditory Pathway: Neuroanatomy Springer-Verlag New York 1992, 66-116.

Costalupes JA, Young ED, Gibson DJ. Effects of continuous noise on rate response of auditory nerve fibers in cat. J. Neurophysiol. 51:1326-1344, 1984.

DAVIS KA. The basic receptive field properties of neurons in the inferior colliculus of decerebrate cats are rarely created by local inhibitory mechanisms. Abstr. Soc. Neurosci. 25:687, 1999.

Davis KA, Ramachandran R, May BJ. Single unit responses in the inferior colliculus of decerebrate cats: II. Sensitivity to interaural level differences. J. Neurophysiol. 82:164-175, 1999.

Delgutte B, JoRIS PX, LITOVSKY RY, YIN TCT. Receptive field and binaural interactions for virtual space stimuli in the cat inferior colliculus. J. Neurophysiol. 81:2833-2851, 1999.

Ehret G, MerZenich MM. Complex sound analysis (frequency resolution, filtering, and spectral integration) by single units of the inferior colliculus of the cat. Brain Res. Rev. 13:139-163, 1988.

FletCHER H. Auditory patterns. Rev. Mod. Phys. 12:47-65 1940.

Gabriele ML, HenKel CK. Organizational patterns of projections from the medial nucleus of the trapezoid body to the lateral superior olivary nucleus. Abstr. Soc. Neurosci. 23:183, 1997.

GAO E, SUGA N. Experience-dependent corticofugal adjustment of midbrain frequency response map in bat auditory system. Proc. Natl. Acad. Sci. 95:12663-12670, 1998.

GiBSON DJ. Interaural crosstalk in the cat. Hear. Res. 7:325-333, 1982.

Gibson DJ, Young ED, Costalupes JA. Similarity of dynamic range adjustment in auditory nerve and cochlear nuclei. J. Neurophysiol. 53:940-958, 1985.

Green D, SWETS J. Signal Detection Theory and Psychophysics Krieger Huntington, NY, 1974.

Jiang D, McAlpine D, Palmer AR. Responses of neurons in the inferior colliculus to binaural masking level difference stimuli measured by rate vs level functions. J. Neurophysiol. 77:30853106, 1997.

KuWAdA S, BAtra R, StAnford TR. Monaural and binaural response 
properties of neurons in the inferior colliculus of the rabbit: effects of sodium pentobarbital. J. Neurophysiol. 61:269-282, 1989.

Lane CC, Delgutte B, Litovsky Ry, Brown MC. Spatial release from masking in the inferior colliculus. Abstr. Soc. Neurosci. 25:687, 1999.

LeBeau Fen, Malmierca MS, Rees A. The role of inhibition in determining neuronal response properties in the inferior collicuIUS. MANLEY GA. Advances in Hearing Research World Scientific Singapore, 1995, 300-313.

LORENTE DE No R. Anatomy of the eighth nerve. III. General plan of structure of the primary cochlear nuclei. Laryngoscope. 43:327-350, 1933.

MAY BJ, SACHS MB. Dynamic range of neural rate responses in the ventral cochlear nucleus of awake cats. J. Neurophysiol. 68:15891602, 1992.

MCFADDEn DM. Masking and the binaural system EngeLs EL. The Nervous System Raven Press, New York, 1975.

MERZENICH MM, REID MD. Representation of the cochlea within the inferior colliculus of the cat. Brain. Res. 77:397-415, 1974.

MOREST DK. The collateral system of the medial nucleus of the trapezoid body of the cat, its neuronal architecture and relation to the olivo-cochlear bundle. Brain Res. 9:288-311, 1968.

MusiCANT AD, ChAN JCK, HIND JE. Direction-dependent spectral properties of cat external ear: new data and cross-species comparisons. J. Acoust. Soc. Am. 87:757-781, 1990.

NARINS MN, EHRET G, TAUTZJ. Accessory pathway for sound transfer in a neotropical frog. Proc. Natl. Acad. Sci. USA 85:1508-1512, 1988.

Oliver DL, Huerta MF. Inferior and superior colliculi. Webster DB, Popper AN, Fay RN. The Mammalian Auditory Pathway: Neuroanatomy Springer-Verlag, New York, 1992, 168-221.

Palmer AR, JiAng D, MCALPIne D. Desynchronizing responses to correlated noise: A mechanism for binaural masking level differences at the inferior colliculus. J. Neurophysiol. 81:722-734, 1999.

Pfalz RKJ. Centrifugal inhibition of afferent secondary neurons in the cochlear nucleus by sound. J. Acoust. Soc. Am. 34:14721477, 1962.

Phillips DP, Calford MB, Pettigrew Jd, Aitkin LM, Semple Mn. Directionality of sound pressure transformation at the cat's pinna. Hear. Res. 8:13-28, 1982.

PHILLIPS DP, CYNADER MS. Some neural mechanisms in the cat's auditory cortex underlying sensitivity to combined tone and widespectrum noise stimuli. Hear. Res. 18:87-102, 1985.

PhILLIPS DP, HaLL SE. Spike-rate intensity functions for cortical neurons studied with combined tone-noise stimuli. J. Acoust Soc. Am. 80:177-187, 1986.

PhILLIPS DP, ORMAN SS, MUSICANT AD, WILSON GF. Neurons in the primary auditory cortex of cats distinguished by their responses to tones and wide spectrum noise. Hear. Res. 18:73-86, 1985.

RamachandRan R, DaVIS KA, MAY BJ. Single-unit responses in the inferior colliculus of decerebrate cats: I. Classification based on frequency response maps. J. Neurophysiol. 82:152-163, 1999.

RAMACHANDRAN R, MAY BJ. ITD sensitivity in the inferior colliculus of unanesthetized decerebrate cats. Abstr. Assoc. Res. O tolar yngol. 22:217, 1999a.

RAMACHANDRAN R, MAY BJ. Sensitivity of inferior colliculus neurons to interaural time differences of amplitude modulated stimuli. Abstr. Soc. Neurosci. 25:688, 1999b.

RatnAm R, Feng AS. Detection of auditory signals by frog inferior collicular neurons in the presence of spatially separated noise. J. Neurophysiol. 80:2848-2859, 1998.

REES A. The coding of intensity in the inferior colliculus: Onset and sustained responses. CaZals Y, Demany L, HoRner K. Advances in the Biosciences, Vol. 83: Auditory Physiology and Perception Pergamon Press, Oxford, 1992, 263-270.

Rees A, Palmer AR. Rate-intensity functions and their modification by broadband noise for neurons in the guinea pig inferior colliculus. J. Acoust. Soc. Am. 83:1488-1498, 1988.

RHOdE WS, GREenBERG S. Lateral suppression and inhibition in the cochlear nucleus of the cat. J. Neurophysiol. 71:493-514 1994.

Rhode WS, Geisler CD, Kennedy DT. Auditory nerve fiber responses to wide-band noise and tone combinations. J. Neurophysiol. 41:692-704, 1978.

Rh Ode WS, OeRTEL D, SMITH PH. Physiological response properties of cells labeled intracellularly with horseradish peroxidase in the ventral cochlear nucleus. J. Comp. Neurol. 213:448-463, 1983.

Rice JJ, MAY BJ, Spirou GA, Young ED. Pinna based spectral cues for sound localization in cat. Hear. Res. 58:132-152, 1992.

RYUGO DK, FEKETE DM. Morphology of primary axosomatic endings in the anteroventral cochlear nucleus of the cat: A study of the endbulbs of Held. J. Comp. Neurol. 210:239-257, 1982.

RYUGO DK, SENTO S. Synaptic connections of the auditory nerve in cats: Relationship between endbulbs of H eld and spherical bushy cells. J. Comp. Neurol. 305:35-48, 1991.

Saberi K, Dostal L, Sadralodabai T, Bull V, Perrot DR. Free field release from masking. J. Acoust. Soc. Am. 90:1355-1370, 1991.

SHOFNER WP, YOUNG ED. Excitatory/ inhibitory response types in the cochlear nucleus: relationships to discharge patterns and responses to electrical stimulation of the auditorynerve. J. Neurophysiol. 54:917-939, 1985.

SCHWARTZ IR. Superior olive and lateral lemniscus. WebSTER DB, Popper RN, FAY AN. The Mammalian Auditory Pathway: Neuroanatomy Springer-Verlag, New York, 1992, 117-167.

SEGEL IH. Biochemical calculations: How to solve mathematical problems in biochemistry. 2nd ed. John Wiley \& Sons, New York, 1976, 214-323.

WINSLOW RL, SACHS MB. Effect of electrical stimulation of the crossed olivocochlear bundle on auditoryner ve responsesto tones in noise. J. Neurophysiol. 57:1002-1021, 1987.

YAN J, SUGA N. Corticofugal amplification of facilitative auditory responses of subcortical combination-sensitive neurons in the mustached bat. J. Neurophysiol. 81:187-824, 1999.

YANG L, Pollak GD, ResLeR C. GABAergic circuits sharpen tuning curves and modify response properties in the mustache bat inferior colliculus. J. Neurophysiol. 68:1760-1774, 1992.

Young ED, BRowneLL WE. Responses to tones and noise of single cells in the dorsal cochlear nucleus of unanesthetized cats. J. Neurophysiol. 39:282-300, 1976.

Young ED, Shofner WP, White JA, Robert J-M, Voigt HF. Response properties of cochlear nucleus neurons in relationship to physiological mechanisms. Edelman GM, GaLL WE, Cowan WM. Auditory Function: Neurobiological bases of Hearing. John Wiley \& Sons, New York, 1988, 277-312. 